\title{
Virtual Network Topology Adaptability based on Data Analytics for Traffic Prediction
}

\author{
Fernando Morales, Marc Ruiz, Lluís Gifre, Luis M. Contreras, Víctor López, and Luis Velasco
}

Invited Paper

\begin{abstract}
The introduction of new services requiring large and dynamic bitrate connectivity can cause changes in the direction of the traffic in metro and even core network segments along the day. This leads to large overprovisioning in statically managed virtual network topologies (VNT), designed to cope with the traffic forecast. To reduce expenses while ensuring the required grade of service, in this paper we propose the VNT reconfiguration approach based on data analytics for traffic prediction (VENTURE); it regularly reconfigures the VNT based on predicted traffic thus, adapting the topology to both, the current and the predicted traffic volume and direction. A machine learning algorithm based on artificial neural network (ANN) is used to provide robust and adaptive traffic models. The reconfiguration problem that takes as input the traffic prediction is modelled mathematically and a heuristic is proposed to solve it in practical times. To support VENTURE, we propose an architecture that allows collecting and storing data from monitoring at the routers and that is used to train predictive models for every origin-destination pair. Exhaustive simulation results of the algorithm together with the experimental assessment of the proposed architecture are finally presented.
\end{abstract}

Index Terms-Virtual Network Topology Reconfiguration, Machine learning for Traffic prediction, Multilayer Networks.

\section{INTRODUCTION}

Qtatic virtual network topologies (VNT), where large $\checkmark$ packet-switching nodes (e.g., IP/MPLS routers) are connected through virtual links (vlinks) supported by static connections in the optical layer, have been commonly designed to cope with the off-net traffic forecast. However, the introduction of cloud infrastructure in the telecom operator' networks to create the telecom cloud [3] facilitates the introduction of new types of service (e.g., live-TV and video distribution [4]), which require large bitrate connectivity and causes changes in the direction of the traffic along the day in metro and even core network segments. This, together with the overall traffic increment that operators' networks are needing to deal with year after year entails that static packet network topologies were largely overprovisioned thus, increasing network total cost of ownership (TCO). In view of that, network operators are looking for more efficient architectures

Manuscript received June 10, 2016.

This work was presented in part at OFC 2016 [1] and [2].

Fernando Morales (fmorales@ac.upc.edu), Lluís Gifre, Marc Ruiz, and Luis Velasco are with the Optical Communications Group (GCO) at Universitat Politècnica de Catalunya (UPC), Barcelona, Spain.

Luis M. Contreras and Victor López are with Telefonica R\&D (TID), Distrito Telefonica, Madrid, Spain. able to reduce TCO, while providing the required grade of service. To that end, VNT need to be dynamically adapted not only to variations in volume, but also to changes in the direction of the traffic.

To support connectivity dynamicity, the IETF has recently standardized the Application-Based Network Operation (ABNO) architecture [5], which includes among others: $i$ ) the ABNO controller as the entrance point to the network for provisioning and advanced network coordination. It acts as a system orchestrator invoking its inner components according to a specific workflow; ii) a Virtual Network Topology Manager (VNTM) in charge of reconfiguring on demand VNT; iii) a Path Computation Element (PCE) to compute the path for new Label Switched Paths (LSP) on the Traffic Engineering Database (TED); iv) a provisioning manager (e.g., a SDN controller) responsible for managing LSPs, both at the optical layer (Lambda Switch Capable, LSC) and at the packet layer (Packet Switch Capable, PSC); and $v$ ) the Operations, Administration, and Maintenance (OAM) Handler to receive notifications and monitored counters.

To automate VNT adaptability, traffic needs to be monitored in the packet nodes and counters be accessible by the OAM Handler. In particular, the disaggregated traffic volume forwarded by each packet node to every other destination node should be available. In addition, notification can be also triggered when the used vlink capacity reaches some configured threshold (e.g., 90\%). In fact, threshold triggered VNT reconfiguration where the OAM Handler receives notifications from the control plane and reroutes individual IP/MPLS paths (PSC LSPs) in a reactive manner was proposed in [6].

However, the number of transponders to be installed tends to be as high as in a static VNT since dimensioning must be done to cope with the maximum of daily traffic forecast during a planning period (e.g., one year). In view of that, we propose an alternative approach to adapt the VNT based on traffic prediction that can reduce the number of transponders to be deployed in scenarios were traffic variations are as a result of changes in the traffic directionality.

Because of its benefits, VNT reconfiguration has been widely studied in the literature. Authors in [7] proposed a centralized path reallocation module running periodically aiming at minimizing the number of used transponders. To follow traffic changes, authors in [8] proposed to add/remove 
one single lightpath each time the VNT is reconfigured. Another topic is using monitored data to produce estimations that can help to anticipate changes in the traffic and proactively reconfiguring the VNT beforehand. In that regard, authors in [9] proposed a method for reducing errors in traffic estimations, while authors in [10] used estimated traffic to predict pre-defined scenarios. Finally, authors in [11] used future traffic estimation to trigger a VNT reconfiguration after the detection of an anomalous amount of traffic between two nodes.

VNT reconfiguration requires from powerful algorithms to analyze large amounts of traffic monitoring data to anticipate, when possible, traffic changes. In this paper, we propose using big data analytics to periodically (e.g., every hour) predict traffic. In case the VNT needs to be reconfigured, predicted traffic is used as input of a VNT optimizer that finds the topology for the next period thus, implementing a decision making process based on the observe-analyze-act loop [11]. Specifically, the contribution of this paper is two-fold:

1) targeting at adapting the VNT to future traffic conditions, in section III we devise a robust and adaptive artificial neural network (ANN) model that is afterwards used as input of the VNT reconfiguration problem; we call this approach as VNT reconfiguration based on traffic prediction (VENTURE). The VENTURE problem is formally stated and formulated as an ILP model in section IV; in view of its complexity, a heuristic algorithm providing better trade-off between complexity and optimality is finally designed;

2) a big data network manager architecture to support VNT adaptability based on traffic prediction from applying data analytics on the monitored traffic data, as well as a workflow assuming the ABNO architecture are proposed in section $\mathrm{V}$.

The discussion is supported by the results from exhaustive simulation over a realistic scenario and from the experimental validation of the big data -based architecture in section VI.

\section{VNT DESIGN AND RECONFIGURATION OptIONS}

As introduced above, several approaches to design and dynamically reconfigure the VNT can be devised. In this section let us first review two of them: $i$ ) the static VNT design and $i$ ) the threshold-based VNT capacity reconfiguration.

In the static VNT design, the topology is designed and dimensioned to cope with the maximum of daily traffic forecast for every origin-destination (OD) pair during a planning period. The resulting topology is thus, capable of supporting the traffic at any time during that period provided a perfect traffic forecast. Fig. 1 presents an example for a sevennode VNT, where the capacity of every vlink supports the maximum daily traffic volume. For illustrative purposes, the plot in Fig. 1a shows the variability in link 6-1 that needs to be dimensioned with a capacity of $200 \mathrm{~Gb} / \mathrm{s}$. Fig. 1b shows the resulting VNT with the capacity of every vlink. It is clear, in view of Fig. 1a that the main drawback of the static VNT design is over-provisioning since most of the available capacity in the VNT will remain underutilized along the day.

To reduce capacity over-provisioning, that of the vlinks can be adapted over time instead of allocating a constant amount of resources. Let us assume that the capacity of the existing vlinks can be increased and decreased to follow the traffic variations but no new vlinks can be created or removed, keeping hence the VNT invariant. Traffic can be monitored at IP routers and when the amount of traffic through a vlink reaches some threshold (e.g., 90\%) the network controller can increase the capacity of such vlink by setting-up a parallel lightpath between the two IP routers; conversely, unused capacity can be released by tearing down lightpaths.

The example in Fig. 2a presents monitored traffic data captured during the last two hours in node 6 , where traffic from that node to every other node in the VNT (labelled as 6$>N$ ), from node 6 to node 7 (6->7), and from node 6 to every other node except to node $7(6->M\{7\})$ are plotted. Fig. $2 b$ shows the initial VNT where every vlink is supported by a 100 $\mathrm{Gb} / \mathrm{s}$ lightpath in the underlying optical layer; the IP/MPLS path for OD 6-7 is also shown. A 90\% threshold is configured and, in the event of threshold violation, the capacity of some vlinks is increased. In our example, two threshold violations for vlinks 1-6 and 1-7 are received, so the VNT capacity is updated (Fig. 2c). It is worth noting that IP/MPLS path for OD 6->7 is not affected by the VNT reconfiguration. As shown in the example, the threshold-based reconfiguration is able to adapt the VNT capacity to traffic changes, so resources in the optical layer are allocated only when vlinks need to increase their capacity. However, the same number of transponders as in the static VNT design approach need to be installed in the IP routers; for instance, in the example in Fig. 2 two transponders are installed in routers 6 and 7 and another four in router 1 reserved for vlinks 1-6 and 1-7.

Let us assume now that, instead of monitoring vlink

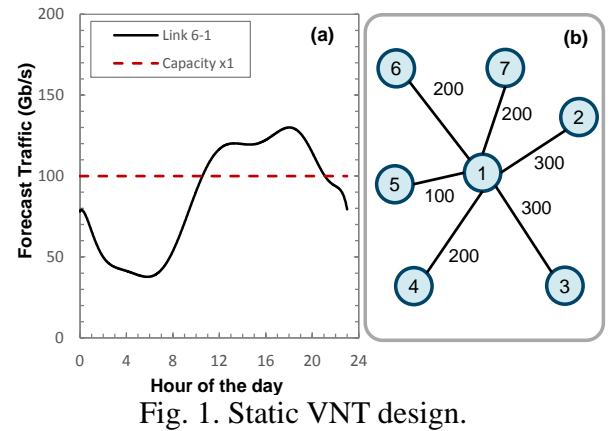

Fig. 1. Static VNT design.

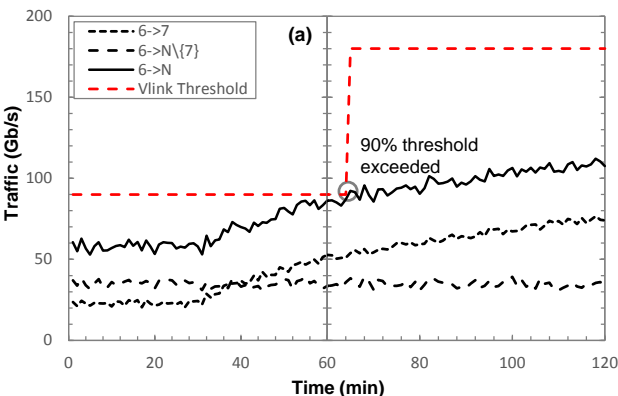

Fig. 2. Threshold-based VNT capacity reconfiguration.

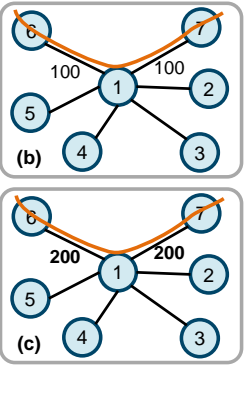

Fig. 3. VNT reconfig.

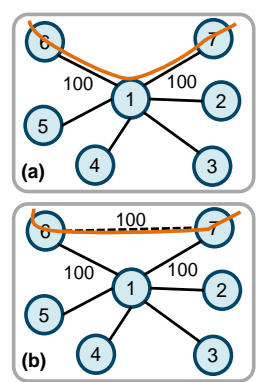


capacity usage, OD traffic is monitored in the routers. Indeed, analyzing the plots in Fig. 2a we realize that traffic 6->7 is responsible for the registered traffic increment. In this case, let us assume that new vlinks can be created/removed in addition to increasing the capacity of the existing ones, so the VNT is actually changed. We propose an approach where OD traffic is periodically analyzed and the current VNT is reconfigured accordingly. An example following this approach is illustrated in Fig. 3, where the OD traffic 6- $>7$ is analyzed at $t=60$ and a maximum value (e.g., $90 \mathrm{~Gb} / \mathrm{s}$ ) is predicted for the next hour. Then, a new vlink between nodes 6 and 7 can be created by establishing a lightpath on the optical layer and traffic 6->7 rerouted (Fig. 3b). Note that this solution reduces two transponders to be installed in router 1 compared to the previous approaches. It is clear that this reduction will happen when the amount of traffic is large enough. In particular, when the amount of traffic exceeds the capacity of the installed transponders (e.g., $100 \mathrm{~Gb} / \mathrm{s}$ ) direct vlinks can be created for part of that traffic, while the residual part could be routed through a different IP/MPLS path.

In order to adapt the VNT to changes in the traffic, we propose a predictive model built upon the monitored OD traffic data. For every OD pair, meaningful statistical values are predicted (e.g., the maximum bitrate for the next hour) and used to adapt the VNT to meet the future traffic matrix, assuming that every OD traffic can be conveyed through two IP/MPLS paths at the most. We call this approach as VENTURE.

\section{DATA ANALYTICS -BASED VNT ADAPTATION}

In this section, we present the proposed modules and the machine learning procedure for the VENTURE approach. We assume that traffic monitoring data is collected at the edge IP routers at regular intervals, e.g., every 15 minutes. Every edge router collects a set of samples for the traffic to every other destination router, which is stored in a collected data repository (Fig. 4). Note that since we focus on OD traffic monitoring, $|N| \cdot(|N|-1)$ traffic samples need to be stored at every monitoring interval, where $|N|$ is the number of routers.

Following a predefined time period, e.g., every hour, a time series from the collected data repository is retrieved for each OD pair and pre-processed applying data stream mining sketches to conveniently summarize collected data thus producing modeled data representing the OD pair that is stored in a modeled data repository. Modeled data includes, among others, for every OD the minimum, maximum, average, and last collected bitrate within the hour.

The set of modeled variables for the current period $t$ is stored in a repository together with variables belonging to previous periods. A prediction module based on machine learning techniques generates the OD traffic matrix predicted for the next period that is used by a decision maker module to decide whether the current VNT needs to be reconfigured. In case that a reconfiguration needs to be performed, the current and the predicted OD traffic matrices are provided to the VNT optimizer to adapt the VNT. Once the algorithm finds a solution, the network controller would be responsible of implementing the changes in the data plane.

The prediction module consists of ANN-based models [12], selected because of its inherent capability of adapting to traffic changes in a non-supervised manner; we consider different ANNs to separately predict the traffic of each OD pair. Each ANN receives as input $p$ previous maximum bitrate measures of the corresponding OD pair from the modeled data repository and returns its expected maximum bitrate at time $t$. Note that considering maximum instead of average bitrate allows adapting the VNT to the maximum expected traffic hence, ensuring a better grade of service.

Since the size of an ANN depends on the number of inputs, hidden layers and neurons, we consider ANN models with $p$ inputs, $s$ neurons in a single hidden layer and one output. Consequently, $s \cdot(p+1)$ coefficients need to be found to specify every ANN. Aiming at keeping the number of coefficients small, we designed the algorithm in Fig. 5 that has to be triggered every time an ANN needs to be refitted. It consists in three phases: $i$ ) input data pre-processing, ii) selection of significant inputs, and iii) dimensioning of the hidden layer.

In the first phase, a time series $X$ with the maximum bitrate for the selected OD pair is retrieved from the modelled data repository. The auto-correlation function (ACF) is applied to $X$ and a list of lags is returned, where the $i$-th lag contains the average correlation between every value in the time series and its $i$-th previous value. Based on the lags analysis, a method is triggered to detect whether a periodic repetitive (seasonal) pattern is observable in $X$ [13]. The resulting period per defines the number of inputs of the ANN; in case of nonseasonal data without observable periodical behavior, we assume per $=24$ (i.e. one day) for convenience. Once per is obtained, $X$ is transformed into a dataset $D$ used for ANN fitting. Every row in $D$ corresponds to a time $t$ within the time series and every column corresponds to a lag within per.

The second phase is an iterative procedure that finds the ANN with the best trade-off between accuracy and number of inputs. This trade-off is captured numerically by the Akaike Information Criterion (AIC) [12]. Starting with $p=p e r$, the ANN routine fits an ANN from dataset $D$ and returns the corresponding AIC value. While the AIC value obtained improves the lowest one obtained so far, the best ANN is stored and $p$ is decremented effectively removing one input. Aiming at reducing the complexity of selecting the input to be removed, we select the lag with lowest ACF. When the minimum AIC is reached, the third phase is executed to increase even more the accuracy of the model by adding hidden neurons until the AIC does not improve. The best ANN is eventually returned.

We face next the VNT reconfiguration (VENTURE) problem to be solved in the VNTM.

\section{THE VENTURE PROBLEM}

In this section, we first formally state the VENTURE 
problem and devise an ILP to model it. In light of the
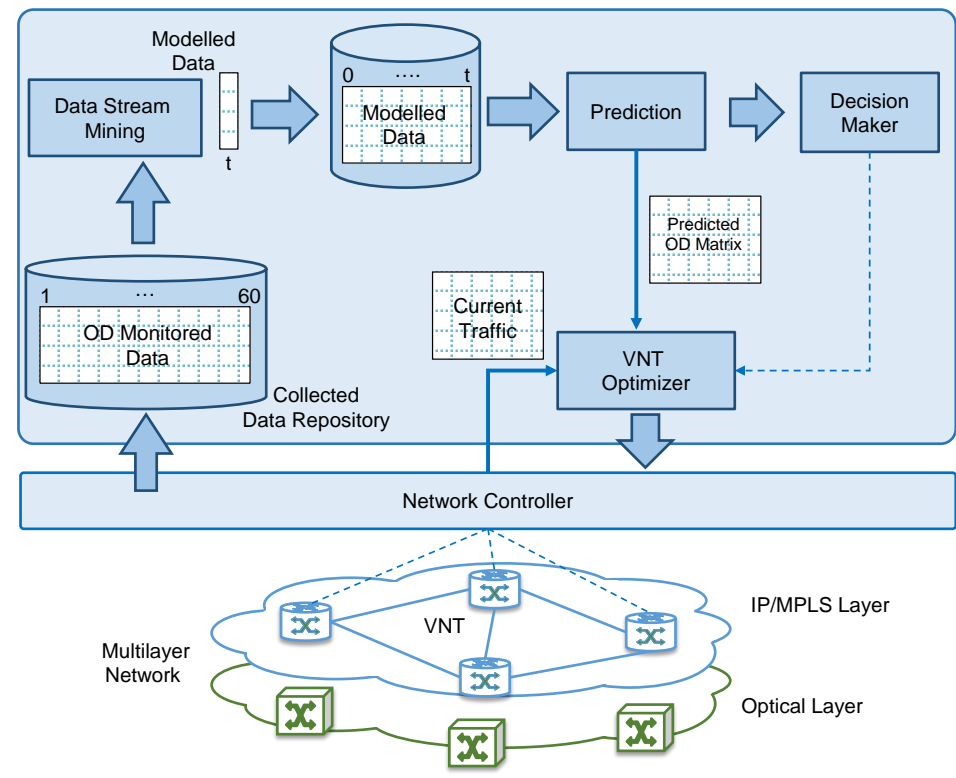

Fig. 4. Applying the observe-analyze-act loop for VNT reconfiguration. complexity of the problem, a heuristic algorithm is eventually devised.

\section{A. Problem Statement}

\section{Given:}

- The current VNT represented by a graph $\mathrm{G}\left(N, E^{\prime}\right)$, being $N$ the set of routers and $E$, Ethe set of current vlinks. Set $E$ is the set of all possible vlinks connecting two routers.

- The set $P$ with the transponders available in the routers; every transponder with capacity $B$.

- The current traffic matrix $D$.

- The predicted traffic matrix $O D$. The bitrate $b_{o}$ of OD pair $o$ must be served following one single path. Only in the case that $b_{o}$ is enough to fill transponders with an amount over a given boundary usage $t b u$, the bitrate of pair $o$ can be split into two flows and served through different paths.

Output: The reconfigured $\operatorname{VNT~G}^{*}\left(N, E^{*}\right)$, where $E^{*} \subseteq E$, and the paths for the traffic on $\mathrm{G}^{*}$.

Objective: Maximize current and predicted served traffic matrices, whilst minimizing the total number of transponders used.

\section{B. Mathematical formulation}

Note from the problem statement that both, the current and the predicted traffic matrices must be served. Consequently, we generate an input traffic matrix OD, where every pair $o$ is the maximum of both, the current and the predicted traffic. In addition, a parameter $k_{o}$ will be used to specify whether pair $o$ can be served using two paths.

The following sets and parameters are defined:

\section{Topology:}

$N \quad$ set of routers, index $n$.

$E \quad$ set of all possible vlinks, index $e$.

$E^{+}(n)$ subset of $E$ with vlinks outgoing from router $n$.

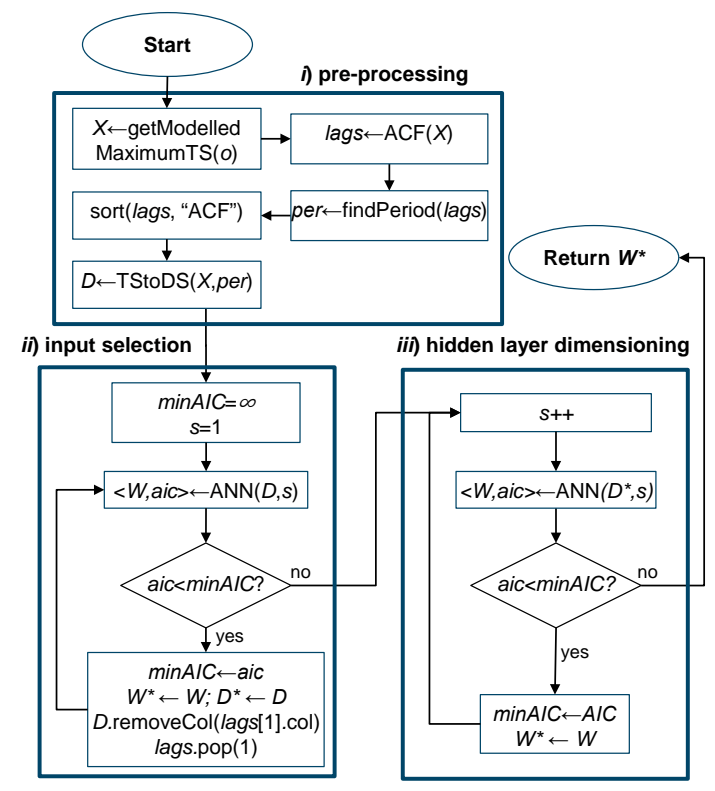

Fig. 5. Self-learning ANN fitting algorithm.

$E^{-}(n)$ subset of $E$ with vlinks incident in router $n$.

Traffic:

$O D$ set of origin-destination pairs, index $o$. Every $o$ is defined by the tuple $<s_{o}, t_{o}, d_{o}, b_{o}>$, where $s_{o}$ and $t_{o}$ specify the source and target nodes, $d_{o}$ the currently served bitrate and $b_{o}$ the maximum of current and predicted bitrate to serve for pair $o$, respectively.

$k_{o} \quad$ maximum number of paths to serve pair $o ; k_{o}=2$ if $b_{o}$ $\geq t b u ; k_{o}=1$ otherwise.

\section{Equipment:}

$P \quad$ set of transponders, index $p$. Every transponder consists of one transmitter (tx) and one receiver (rx).

$P^{+}(n)$ subset of tx transponders in router $n$.

$P^{-}(n) \quad$ subset of rx transponders in router $n$.

$P(n) \quad$ subset of transponders in $n . P(n)=P^{+}(n) \cup P^{-}(n)$.

$B \quad$ capacity of every transponder.

The decision variables are:

$x_{p} \quad$ binary, 1 if transponder $p$ is used, 0 otherwise.

$x_{p e}$ binary, 1 if transponder $p$ is used to support vlink $e, 0$ otherwise.

$x_{o k} \quad$ integer, fraction of bitrate of pair $o$ served through path $k$.

$x_{o k e}$ integer, fraction of bitrate of pair $o$ served through path $k$ using vlink $e$.

$z_{o k e}$ binary, 1 if pair $o$ is routed using path $k$ through vlink $e$, 0 otherwise.

$y_{n} \quad$ integer+, number of transponders used at router $n$.

$v_{o} \quad$ integer+, fraction of unserved bitrate of pair $o$.

Then, the proposed ILP formulation is as follows:

$\min (|P|+1) \cdot \sum_{o \in O D} v_{o}+\sum_{n \in N} y_{n}$

subject to: 


$$
\begin{aligned}
& \sum_{e \in E^{+}(n)} z_{o k e}-\sum_{e \in E^{-}(n)} z_{\text {oke }}= \begin{cases}1 & \forall o \in O D, k=1 . . k_{o}, n=s_{o} \\
0 & \forall o \in O D, k=1 . . k_{o}, \\
-1 & n \in N \backslash\left\{s_{o}, t_{o}\right\} \\
& \forall 0 \in O D, k=1 . . k_{o}, n=t_{o}\end{cases} \\
& x_{\text {oke }} \leq b_{o} \cdot z_{\text {oke }} \quad \forall o \in O D, k=1 . . k_{o}, e \in E \\
& x_{o k e} \leq x_{o k} \quad \forall o \in O D, k=1 . . k_{o}, e \in E \\
& x_{o k}-b_{o} \cdot\left(1-z_{o k e}\right) \leq x_{o k e} \quad \forall o \in O D, k=1 . . k_{o}, e \in E \\
& \sum_{k=1}^{k_{o}} x_{o k}+v_{o} \geq b_{o} \quad \forall 0 \in O D \\
& \sum_{k=1}^{k_{o}} x_{o k} \geq d_{o} \quad \forall o \in O D \\
& \sum_{o \in O D} \sum_{k=1}^{k_{o}} X_{o k e} \leq B \cdot \sum_{p \in P^{+}(i)} X_{p e} \quad \forall e=(i, j) \in E \mid i, j \in N \\
& \sum_{o \in O D} \sum_{k=1}^{k_{o}} x_{o k e} \leq B \cdot \sum_{p \in P^{-}(j)} x_{p e} \quad \forall e=(i, j) \in E \mid i, j \in N \\
& \sum_{e \in E^{+}(n)} x_{p e} \leq x_{p} \quad \forall n \in N, p \in P^{+}(n) \\
& \sum_{e \in E^{-}(n)} x_{p e} \leq x_{p} \quad \forall n \in N, p \in P^{-}(n) \\
& \sum_{p \in P^{+}(n)} x_{p} \leq y_{n} \quad \forall n \in N \\
& \sum_{p \in P^{-}(n)} x_{p} \leq y_{n} \quad \forall n \in N
\end{aligned}
$$

The multi-objective cost function (1) minimizes both, unserved traffic and used transponders, where the highest cost corresponds to the first term.

The network flow constraints in (2) define paths on the topology for every OD pair. Each of these paths has a continuous capacity assignment along its route, as imposed by constraints (3)-(5). Notwithstanding constraint (6) allows serving only a fraction of the total capacity $b_{o}$ of every OD pair; that fraction has to include at least the currently served bitrate as stated in constraint (7). Note that optimal solutions might include loops that they can be safely removed in a postprocessing phase.

Constraints (8)-(13) deal with transponder equipment. Constraints (8) and (9) assign transmission and reception transponders to vlinks, respectively to support the capacitated paths. Constraints (10) and (11) prevent from assigning one transponder to multiple vlinks. Finally, constraints (12) and (13) compute the maximum between the number of transponders used for transmission and reception at every node, which represents the number of transponders to be installed in every router.

ILP problems belong to the NP-complete complexity class, as proven in [14]. The size of the proposed formulation is $\mathrm{O}\left(|N|^{4}+|P| \cdot|N|^{2}\right)$ variables and $\mathrm{O}\left(|N|^{4}+|P| \cdot|N|\right)$ constraints. As an example, the size of the above formulation for the network instance with $k_{0}=2$ and 14 nodes presented in section VI is of $2 \cdot 10^{5}$ variables and $10^{5}$ constraints. As a result, solving the proposed formulation becomes impractical for realistic scenarios even using commercial solvers; in our tests, solving times were longer than $10 \mathrm{~h}$. Consequently, we developed a heuristic algorithm that provides much better trade-off between optimality and complexity.

\section{Heuristic algorithm}

We devise a heuristic algorithm to solve the VENTURE problem consisting of three phases; the pseudocode is presented in Table I. After deallocating current traffic and releasing used resources (lines 2-5 in Table I), bitrate $b_{o}$ of OD pairs is split into two different flows and stored in set $Q$ : flow $g_{o}$ carries bitrate enough to fill transponders with an amount over tbu and flow $l_{o}$ carries the remaining bitrate; these flows will be routed through different paths (lines 6-7). Next, the first two phases focus on routing every flow $g_{0}$ through the direct vlink connecting source and destination routers (lines 89); sets $F$ stores the path of the flows. The first phase selects those flows for which a direct vlink already exists in the current VNT and the second phase does the same for the rest of flows thus, creating new direct vlinks. After these two phases, the residual bitrate $u_{o}$ is checked and stored in set $U$. If all traffic has been already served, the algorithm ends (lines 10-12); otherwise, OD pairs are sorted by the amount of unserved bitrate and the third phase eventually routes the unserved bitrate by possibly increasing the capacity of existing vlinks or by adding new ones (line 13). The reconfigured VNT and the new routing are eventually returned.

The algorithm for the first phase is detailed in Table II. The uncapacitated original topology is used to route flows $g_{o}$ through an existing direct vlink so as to introduce inertia to the changes in the current topology (line 3 in Table II). The number of transponders to be allocated in the end routers of the direct vlink is computed as the minimum between the amounts of transponders needed to allocate $g_{o}$ and the unused transponders (lines 4-7); those transponders are allocated to

TABLE I MAIN ALgORITHM

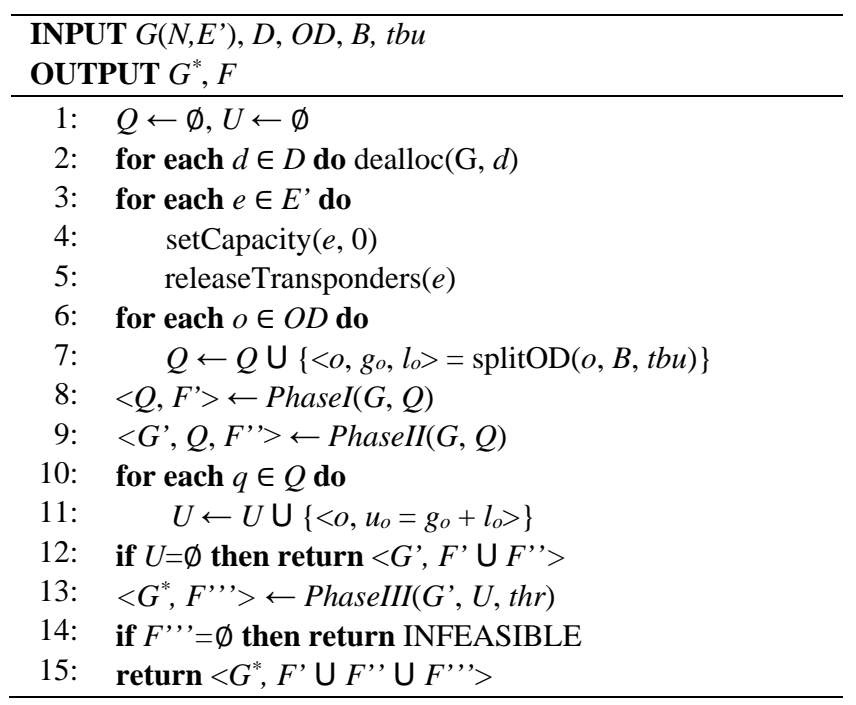


TABLE II PHASEI ALGORITHM

\begin{tabular}{rc} 
INPUT $G(N, E), Q$ \\
OUTPUT $Q, F$ \\
\hline 1: $\quad F \leftarrow \emptyset$ \\
2: $\quad$ for each $q=<0, g_{o}, l_{o}>\in Q$ do \\
3: $\quad$ if $e=\left(s_{o}, t_{o}\right) \notin E$ OR $g_{o}=0$ then continue \\
4: & $n_{o} \leftarrow$ ceil $\left(g_{o} / B\right)$ \\
$5:$ & $n_{s} \leftarrow$ getNumUnusedTransponders $\left(s_{o}, P^{+}\right)$ \\
6: & $n_{t} \leftarrow$ getNumUnusedTransponders $\left(t_{o}, P^{-}\right)$ \\
7: & $n \leftarrow \min \left\{n_{o}, n_{s}, n_{t}\right\}$ \\
8: & allocateTransponders $(e, n)$ \\
9: & $f \leftarrow \operatorname{SP}\left(G, o, g_{o}\right)$ \\
10: & if $f \neq \emptyset$ then \\
11: & allocate $(G, f)$ \\
12: & $F \leftarrow F \cup\{f\}$ \\
13: & $g_{o} \leftarrow g_{o}-f . b$ \\
$14:$ & return $<Q, F>$
\end{tabular}

add capacity to the direct vlink (line 8) and a shortest path is computed on the resulting VNT (line 9). In case that a path is found (i.e., capacity was added to the direct vlink), the path is allocated and the amount of served bitrate reduced from the one requested (lines 10-13). The updated set $Q$ and the found paths stored in set $F$ are eventually returned (line 14).

The second phase is similar to the first phase, but for flows $g_{o}$ through non existing direct vlink. New capacitated direct vlinks are thus, added to the topology to support those flows.

In the third phase, the current topology is extended to a full mesh topology by adding uncapacitated vlinks (lines 2-4 in Table III). Next, a randomized routing procedure is run for a given number of iterations (lines 6-31); at every iteration, the initial extended topology and the unserved bitrate are cloned and the latter randomly sorted, giving higher priority to flows with higher remaining traffic (lines 7-12). Those flows with unserved bitrate are routed using one single path (lines 13-16). Aiming at minimizing the number of used transponders, link metrics are set proportional to the number of transponders needed to allocate the remaining capacity of the current flow (line 15). If no path is found, the corresponding cost is added to the iteration cost (lines 17-19); otherwise, in case the path capacity does not serve the remaining bitrate, we check whether the capacity of its links can be increased using the available resources, i.e., transponders in the end nodes and spectral resources to create a lightpath at the optical layer (lines 20-22). Finally, the path is allocated and the remaining bitrate of the flow is updated, as well as the iteration cost (lines 23-26).

Once a solution has been built, a local search procedure is executed (line 27) aiming at finding a local minimum. The best topology and the found paths are returned as final solution (lines 29-32).

The local search procedure tries to reduce the total number of used transponders during the constructive phase. Since the number of transponders used in a node is computed as the maximum between transmission and reception, this procedure focuses on releasing transponders that actively contribute to
TABLE III PhASEIII ALgORITHM

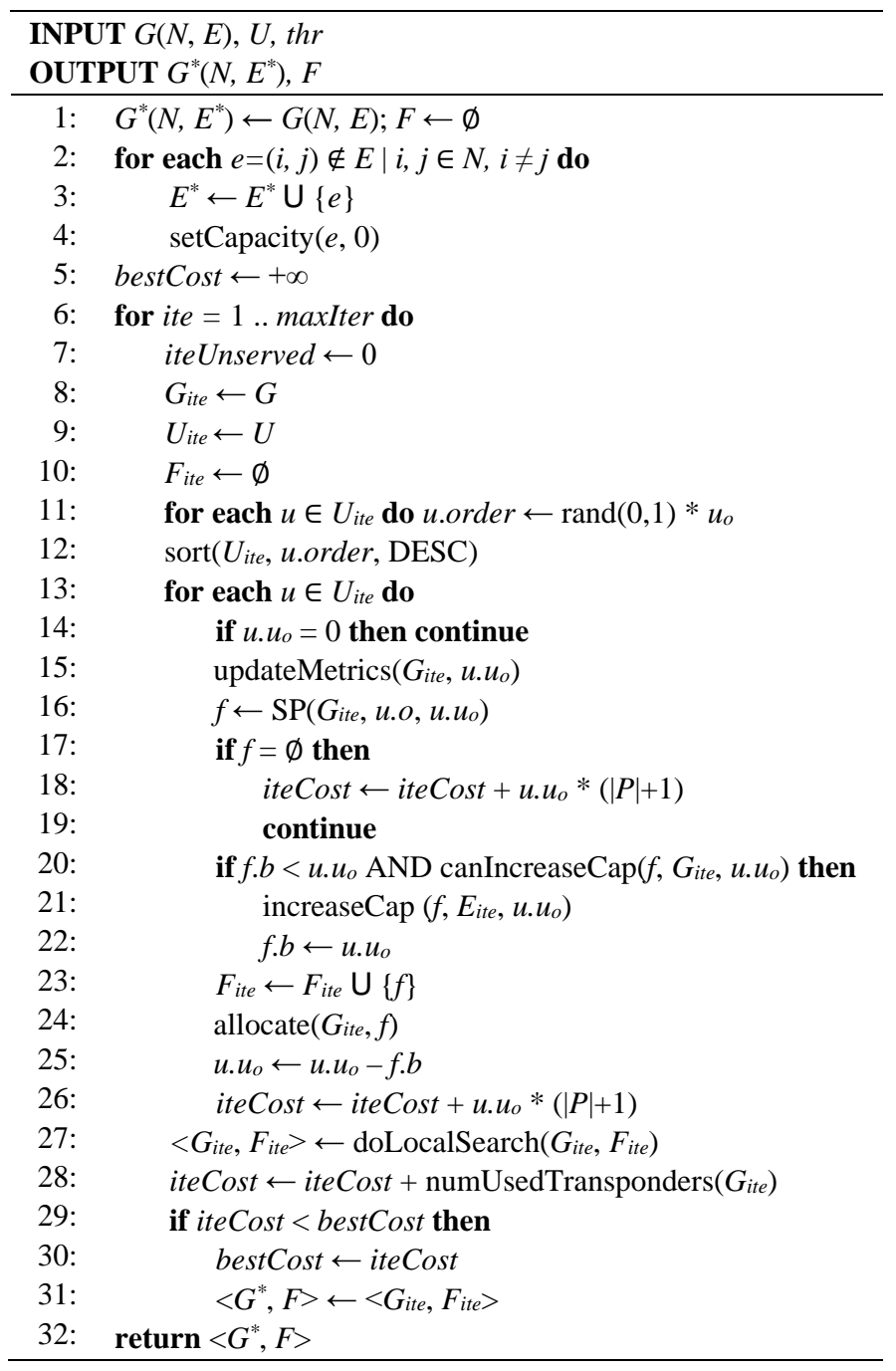

Table IV Local Search Procedure

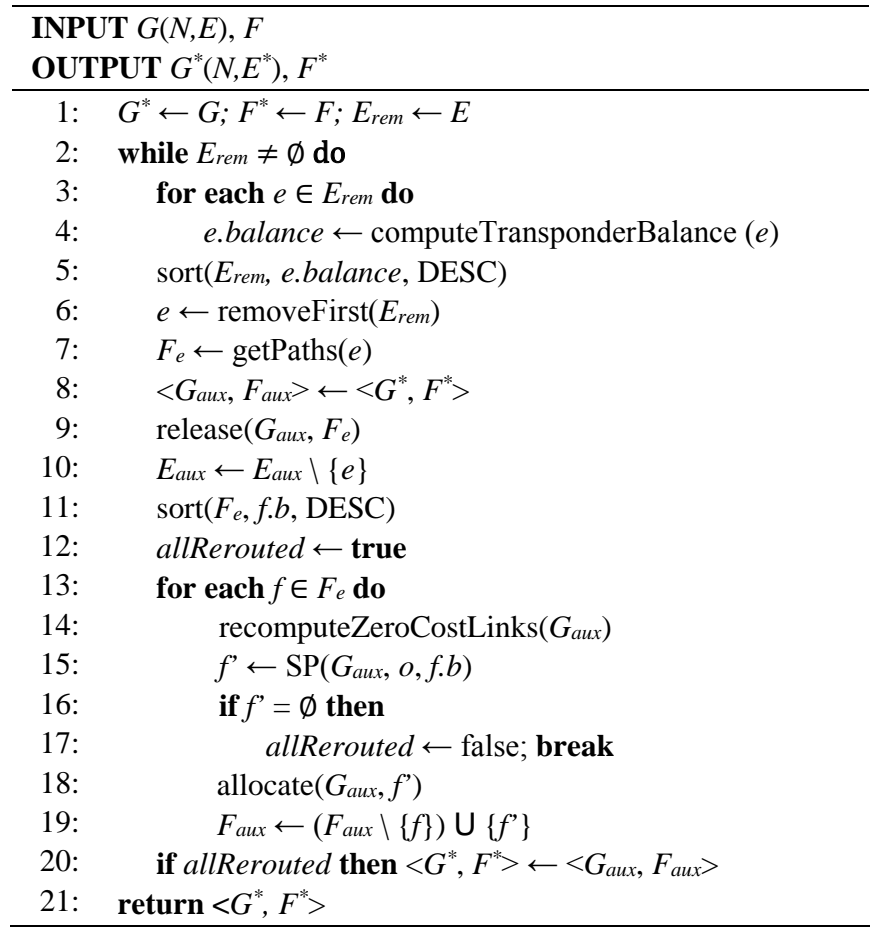




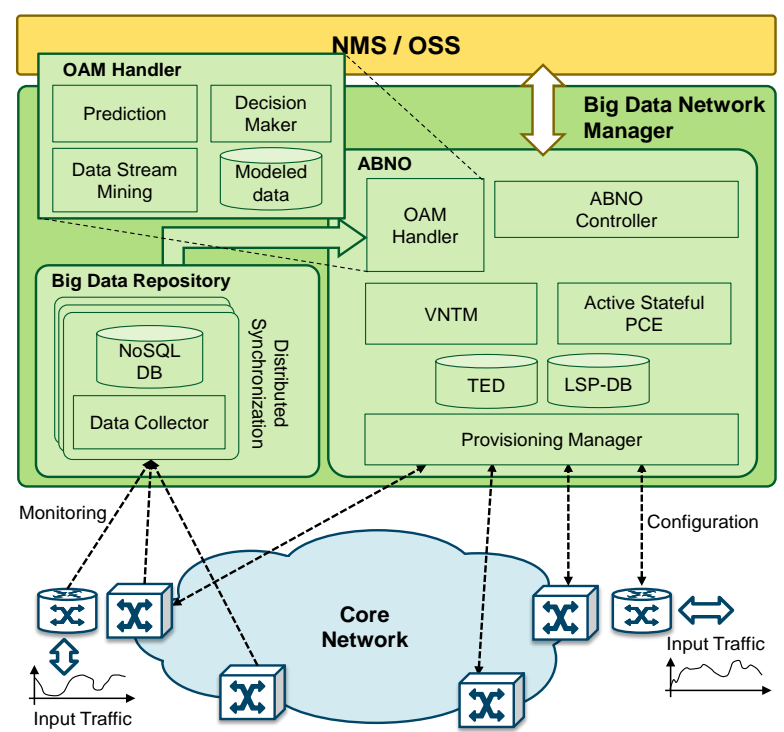

Fig. 6. Big data network manager architecture.

that maximum at every node. The algorithm is detailed in Table IV, where all current vlinks in the VNT are processed (lines 2-20). The vlink with ports most actively contributing to the cost function is selected along with the set of paths routed through it (lines 6-7). This set is released from the VNT and sorted with respect to the bitrate (lines 8-11). Next, the set of paths is re-routed by possibly using new vlinks at zero objective cost (lines 13-15). In case of a feasible solution, the VNT is updated with these changes (line 20).

Table $\mathrm{V}$ presents the time complexity of the proposed algorithms, where $R 0$ is the worst case time complexity to find a feasible lightpath to support every direct vlink. For illustrative purposes, the computation time for the scenario with 14 nodes presented in section VI is also provided.

TABle V Time CompleXity of the Algorithms

\begin{tabular}{ccc}
\hline Phase I/II & Phase III (per iter) & Local Search \\
\hline $\mathrm{O}\left(|N|^{2} \cdot R 0\right)$ & $\mathrm{O}\left(|N|^{2} \cdot(|E| \cdot \log |N|+R O)\right)$ & $\mathrm{O}\left(|E|^{2} \cdot(\log |E|+|F e| \cdot \log |N|)\right)$ \\
$<1 \mathrm{~m}$ & $298 \mathrm{~ms}$ & $234 \mathrm{~ms}$ \\
\hline
\end{tabular}

\section{Proposed Architecture ANd Workflow}

To support the generic modules introduced in section III for VENTURE, we propose the architecture in Fig. 6. A big data repository consisting of a distributed database and a data collector is used to store collected data. ABNO's OAM Handler includes data stream mining sketches, the modeled data repository, the prediction module running the proposed ANN to anticipate next period traffic conditions and the decision maker that decides whether the VNT should be updated based on the predicted traffic. Finally, ABNO's VNTM is in charge of computing the new VNT.

Fig. 7 presents the proposed workflow. Monitored traffic data is sent to the collected data repository ready to be analyzed. Periodically, ABNO's OAM Handler retrieves aggregated monitored data and applies data stream mining techniques on the received data to transform monitored data

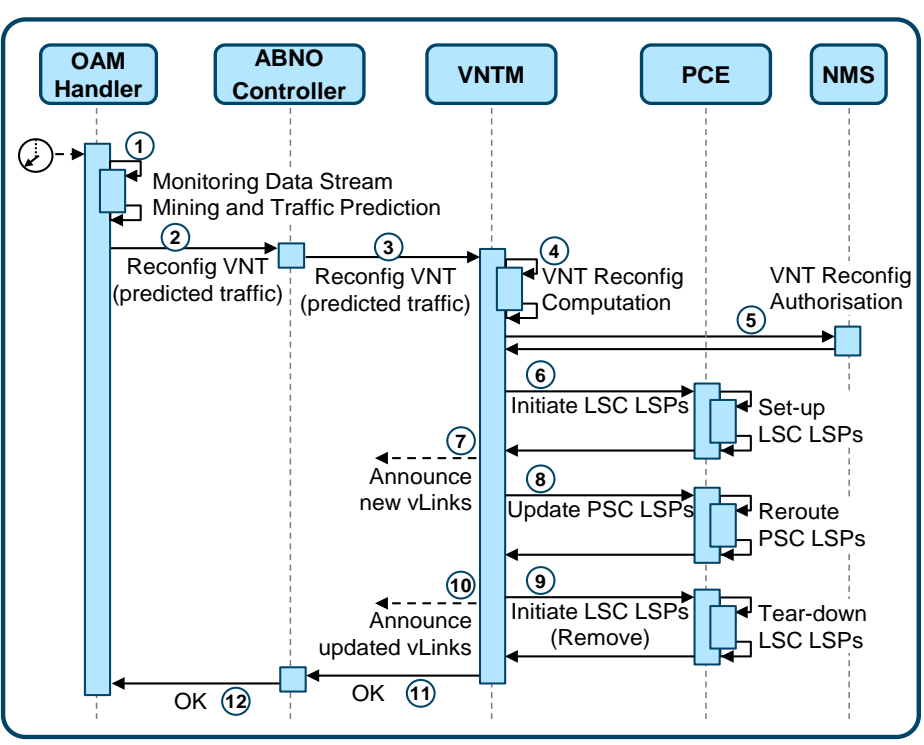

ig. 7. Proposed workflow.

into modeled data. Modeled data is used by the prediction module, running the proposed ANN (labeled as 1 in Fig. 7). Based on the predicted traffic, a decision maker module decides whether the VNT should be updated. In case of VNT reconfiguration, the VNTM is in charge of computing the new VNT. To that end, the OAM Handler issues a request to the ABNO controller that includes the predicted traffic together with some other parameters to facilitate VNTM computation (2) and the ABNO controller initiates the workflow forwarding the request to the VNTM (3).

The VNTM computes the new VNT with the predicted traffic matrix received from the OAM Handler (4). Continuing with our seven-node VNT example, let us assume that the new VNT consists on adding the new virtual link 6-7 and reducing the capacity of some other vlinks. The solution is first notified to the NMS (5) and then, its implementation is divided into a sequence to avoid traffic disruption as anticipated above: firstly, lightpath (LSC LSP) 6-7 is created (6) and the new vlink is advertised (7); next, PSC LSPs are rerouted (8) (a make-before-brake strategy to avoid disruption can be implemented) and unused capacity in vlinks 1-3 and 1-4 removed by tearing down the underlying LSC LSPs (9); new vlinks' capacity is advertised (10). Upon VNT reconfiguration completion, VNTM replies the ABNO controller (11), which eventually replies the OAM Handler (12).

\section{ILLUSTRATIVE RESULTS}

This section focuses first on validating the VENTURE approach through simulation. Next, the proposed architecture is experimentally assessed in our SYNERGY testbed.

\section{A. Simulation results}

For evaluation purposes, we implemented an event-driven simulator in OMNeT++ containing the modules described in Fig. 4. To measure the effect of volumetric and directional changes in traffic, we implemented generators that inject traffic following two pre-defined traffic profiles named as 

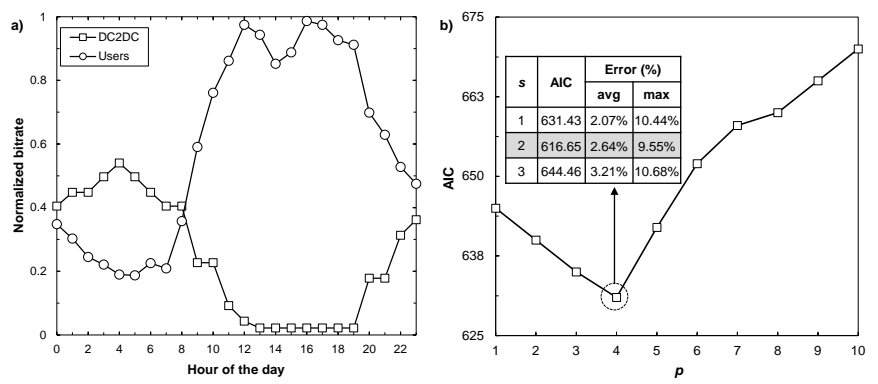

Fig. 8. Average daily traffic profiles used in the simulation (a) and ANN goodness-of-fit (b).

Users and Datacenter-to-Datacenter (DC2DC) (see average daily evolution in Fig. 8a). In addition, some random values around the average value are usually observed in real traffic. In consequence, a function $\varepsilon_{t}$ representing random variable traffic is also added. We assume that $\varepsilon_{t}$ follows a 0 -centered normal (Gaussian) probability distribution, i.e., $\varepsilon_{t} \sim N\left(0, \sigma^{2}\right)$ where $\sigma$ represents the standard deviation. In consequence, the daily traffic profile of every OD pair can be defined as $Y_{O D}(t)$ $=\alpha \cdot f(t)+\varepsilon_{t}$, where function $f(t)$ represents the average traffic profile and $\alpha$ is a scaling factor in $\mathrm{Mb} / \mathrm{s}$.

Finally, the set of nodes was divided into two subsets to generate changes in the direction of the traffic; ODs with destination one of the nodes in the first subset follow the Users profile, while the others follow the $D C 2 D C$ one. We consider a scenario where a maximum of $26 \times 100 \mathrm{~Gb} / \mathrm{s}$ transponders per node are equipped. With such configuration, the static and threshold-based approaches are applied to a full-
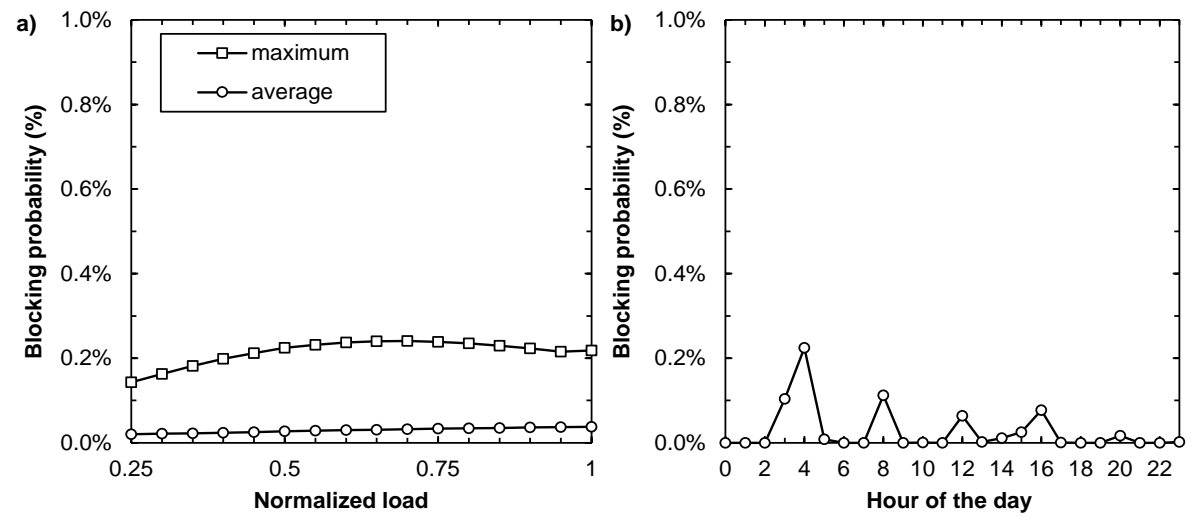

Fig. 9. Average and maximum hourly blocking prob. of VENTURE vs. load (a). Blocking prob. along one day and for normalized loads 0.48 (b) and 1.0 (c).
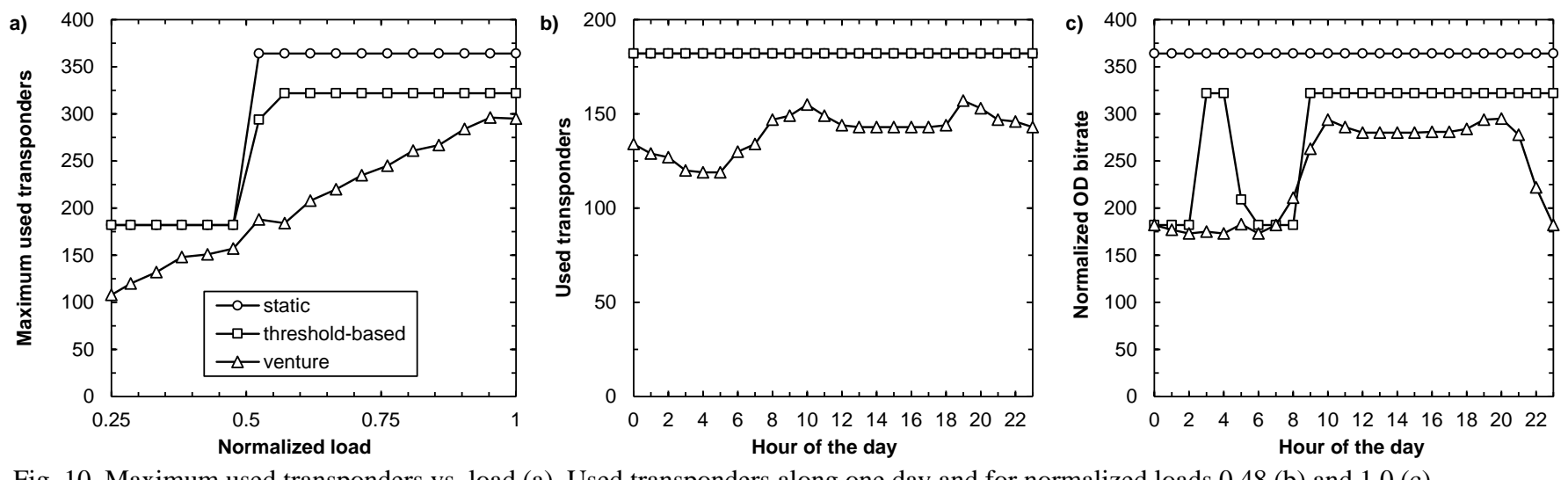

Fig. 10. Maximum used transponders vs. load (a). Used transponders along one day and for normalized loads 0.48 (b) and 1.0 (c).

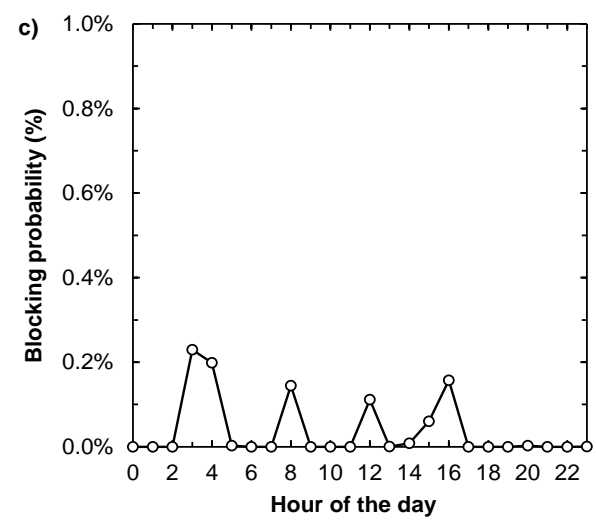

mesh 14-node VNT, where the initial capacity of each vlink

The ANN models are trained applying the fitting algorithm in Fig. 5 on a training dataset with modelled data belonging to the last week. Results in Fig. 8b illustrate the average size and goodness-of-fit of ANN models. Recall that during the input selection phase, the number of inputs $p$ is decreased aiming at minimizing the AIC value. We observe that the minimum AIC is on average reached at $p=4$, being mainly selected those inputs from $t-1$ to $t-4$. Results from the hidden layer dimensioning phase are shown in the table embedded in Fig. $8 b$, for a number of hidden neurons ranging from 1 to 3 . Note at the minimum AIC is obtained for $s=2$, which results in an $\mathrm{N}$ model with 10 coefficients that accurately predicts the maximum relative errors ( $2.64 \%$ and $9.55 \%$, respectively).

Next, we compare the effect in the unserved traffic and the number of used transponders under the threshold-based approach (we assumed $90 \%$ threshold) that runs continuously and under the VENTURE one that it is triggered at fixed intervals of one hour. For the sake of completeness, the static case where no reconfiguration is performed is also included.

Fig. 9 presents the obtained blocking probability for the range of loads considered; values for both, the static and the threshold-based approaches are omitted since yield zero blocking probability. In the case of the VENTURE approach, Fig. 9a plot the average and maximum hourly blocking along a ranges from 100 to $200 \mathrm{~Gb} / \mathrm{s}$. 
day. We observe that, for a wide range of traffic loads, maximum blocking probability is below $0.24 \%$, while that on average is virtually zero. Fig. 9b-c analyze the evolution of blocking probability during the day for the lowest and highest load, respectively. We observe that small peaks of blocking probability appear related to abrupt changes in the injected traffic and last for a couple of hours at the most, which is the time that VENTURE takes in fully adapting the VNT to traffic changes with the specific configuration selected.

Fig. 10 focuses on the use of transponders. Fig. 10a plots the maximum transponder usage as a function of the load for each approach. Both, the static and the threshold-based approaches show a constant transponder usage for loads lower than 0.5, which is increased from that load up. For low loads, the capacity of vlinks in the fully meshed VNT is $100 \mathrm{~Gb} / \mathrm{s}$ in both cases and it is increased to $200 \mathrm{~Gb} / \mathrm{s}$ for high loads under the static approach. The threshold-based approach, however, is able to manage the use of transponders by flexibly using available transponders to increment the capacity of vlinks running out of capacity; this way it achieves transponder savings up to $11 \%$ with respect to the static VNT approach.

Interestingly, transponder usage scales linearly with the load with VENTURE. Compared to the threshold-based approach, VENTURE obtains savings between $8 \%$ and $42 \%$.

Fig. 10b-c focus on the use of transponders along the day for the lowest and highest loads for the three approaches. Apart from the constant transponder usage in the static approach, we show the different usages of the threshold-based and the VENTURE approaches. In particular, we observe how the VENTURE approach is able to remarkably reduce up to $45 \%$ transponder usage at some hours, mainly when the DC2DC traffic profile is dominant. On the other hand, in those hours when Users traffic profiles dominate, transponder usage under VENTURE still outperforms that of the threshold-based approach.

In conclusion, the VENTURE approach maximizes the overall utilization of available transponders in two different ways: $i$ ) by reconfiguring the virtual topology to follow traffic direction changes, and ii) by increasing the capacity of vlinks when the traffic increases.

\section{B. Experimental assessment}

Experiments have been carried out on the UPC's SYNERGY test-bed. Apache Cassandra database [15] was used as a big data repository and a data collector module was implemented to offer an UDP- based interface to the monitors, storing the received data in Cassandra. Apache Spark [16] was used to implement data stream mining and machine learning techniques. Finally, ABNO modules in Fig. 6 were implemented using UPC's iONE software [17]. A HTTP REST API interface was implemented between the OAM Handler and the ABNO controller and from it to VNTM, so as to report the predicted traffic matrix. PCEP was used between VNTM, PCE, and the provision manager. Finally, BGP-LS was used to synchronize TEDs. In particular, VNTM is in charge of advertising topological changes in the VNT, including vlink creation and releasing, as well as updating vlink capacity changes.

Fig. 11 illustrates monitored traffic data being periodically

\begin{tabular}{|c|c|c|c|c|c|}
\hline $\begin{array}{l}\nabla \text { Monitors Data } \\
\text { Message Type: } 1\end{array}$ & No. & Time & Source & Destin & Info \\
\hline Message Length: 88 & 391 & 58.070 & 172.16 .103 .103 & Datac & Monitor Data from Source 172.16.103.103 \\
\hline Source: 172.16 .103 .106 & 392 & 58.070 & 172.16 .103 .107 & Datac & Monitor Data from Source 172 \\
\hline TimeStamp: 1441138660412471 & 393 & 59.065 & 172.16 .103 .101 & DataCo & Monitor Data from Source 172. \\
\hline$\checkmark$ TLV Monitored BitRate & 394 & 59.066 & 172.16 .103 .104 & DataCollec & Monitor Data from Source 172 \\
\hline & 395 & 59.066 & 172.16 .103 .106 & DataCollec & Monitor Data from Source 172.16.103.106 \\
\hline $\begin{array}{l}\nabla \text { TLV Monitored BitRate } \\
\text { Destination: } 172.16 .103 .107\end{array}$ & 396 & 59.069 & 172.16 .103 .105 & DataCollec & Monitor Data from Source 172.16.103.105 \\
\hline BitRate: $51100 \mathrm{Mb} / \mathrm{s}$ & 397 & 59.070 & 172.16 .103 .102 & DataCollec & Monitor Data from Source 172.16.103.102 \\
\hline$\checkmark T L V$ Monitored BitRate & 398 & 59.071 & 172.16 .103 .103 & DataCollec & Monitor Data from Source 172.16.103.103 \\
\hline$\triangleright$ TLV Monitored BitRate & 399 & 59.072 & 172.16 .103 .107 & DataCollec & Monitor Data from Source 172.16.103.107 \\
\hline DTLV Monitored BitRate & 403 & 91 & OAMHandler & Cassandra & GET /3af90b/monitorsData?ti=1..512\&tj=1.. \\
\hline & 411 & 59.754 & Cassandra & OAMHandler & (application/ison) \\
\hline
\end{tabular}

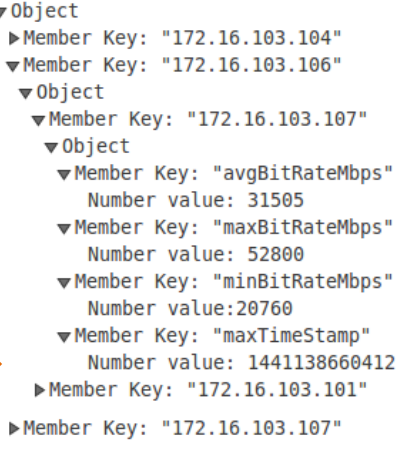

Fig. 11. Exchanged messages for monitored traffic Collection.

\begin{tabular}{|c|c|c|c|c|c|c|}
\hline & No. & Time & Source & Destin & Protocol & Info \\
\hline & 465 & *REF* & OAMHandler & ABNOCtrl & HTTP/XML & POST /ctrl/VNTReconfig HTTP/1.0 \\
\hline & 468 & 0.000 & ABNOCtrl & VNTManager & HTTP/XML & POST /vntm/VNTReconfig HTTP/1.0 \\
\hline & {$[471$} & 0.028 & VNTManager & NMS & HTTP/XML & POST /nms/VNTReconfig HTTP/1.0 \\
\hline & 475 & 0.030 & NMS & VNTManager & HTTP/XML & HTTP/1.1200 OK \\
\hline & 478 & 0.030 & VNTManager & PCE & PCEP & Path Computation LSP Initiate (PCInitiate) \\
\hline & 483 & 0.076 & PCE & VNTManager & PCEP & Path Computation LSP State Report (PCRpt) \\
\hline & {$[484$} & 0.077 & VNTManager & PCE & BGP & UPDATE Message \\
\hline & 486 & 0.082 & VNTManager & PCE & BGP & UPDATE Message \\
\hline & {$[495$} & 0.097 & VNTManager & PCE & PCEP & Path Computation LSP Update Request (PCUpd) \\
\hline & 497 & 0.104 & PCE & VNTManager & PCEP & Path Computation LSP State Report (PCRpt) \\
\hline & 498 & 0.104 & VNTManager & PCE & PCEP & Path Computation LSP Initiate (PCInitiate) \\
\hline & 499 & 0.104 & VNTManager & PCE & PCEP & Path Computation LSP Initiate (PCInitiate) \\
\hline & 500 & 0.104 & VNTManager & PCE & BGP & UPDATE Message \\
\hline & 503 & 0.109 & VNTManager & PCE & BGP & UPDATE Message \\
\hline & 509 & 0.167 & PCE & VNTManager & PCEP & Path Computation LSP State Report (PCRpt) \\
\hline & 511 & 0.216 & PCE & VNTManager & PCEP & Path Computation LSP State Report (PCRpt) \\
\hline & 513 & 0.217 & VNTManager & ABNOCtrl & HTTP/XML & HTTP/1.0 $2000 \mathrm{~K}$ \\
\hline (12) & 515 & 0.217 & ABNOCtrl & OAMHandler & HTTP/XML & HTTP $/ 1.0200$ OK \\
\hline
\end{tabular}

Fig. 12. Exchanged messages for VNT reconfiguration.

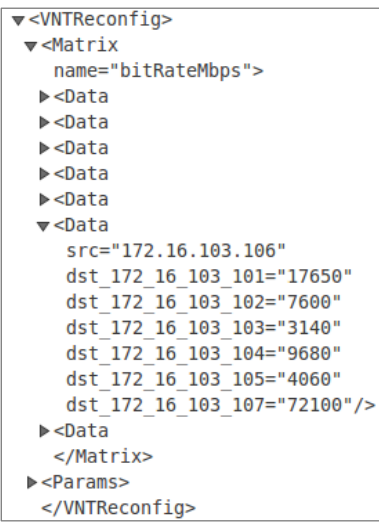

Fig. 13. Message (2) details. 
send by the packet nodes to the data collector, as well as the request that the OAM Handler issues to Cassandra's REST API to collect monitored data. UDP monitoring messages contain, among others, the source node and the timestamp of the sample, and for each aggregated flow leaving the node to a destination, its destination node and bitrate. After selecting and aggregating monitored data between the selected times $t_{i}$ and $t_{j}$, Cassandra replies with a JSON-encoded matrix specifying for each pair of source-destination the average, maximum and minimum bitrate

Fig. 12 shows the meaningful messages exchanged between ABNO modules. For the sake of clarity, messages are identified following the workflow in Fig. 6. The OAM handler sends a REST API request to the ABNO controller (message 2) containing the predicted traffic matrix for the next period. The details of that message are presented in Fig. 13. After receiving the predicted traffic matrix, the VNT computes the optimal VNT and issues requests to the PCE to implement the LSC LSPs supporting the new vlinks, reroute the selected PSC LSPs, and tear down unused LSC LSPs. In addition, VNT changes are advertised to the rest of ABNO modules. The total process took $217 \mathrm{~ms}$, from the instant the OAM handler triggered the workflow.

\section{CONCLUSIONS}

An efficient approach, named as VENTURE, to adapt the current VNT to future traffic conditions aiming at minimizing TCO has been proposed. The approach consists in monitoring OD traffic in the IP/MPLS routers and applying data analytics to learn predictive models that are used as inputs of a reconfiguration problem. In particular, an ANN for every OD pair was proposed as a predictive model along with an algorithm to obtain a highly accurate ANN using as few coefficients as possible. The VENTURE reconfiguration problem was formally stated and formulated as an ILP. In view of its complexity for short-term valid solutions, a heuristic algorithm to provide near optimal solutions in practical computation times was proposed.

In addition, a big data analytics OAM handler has been proposed to support VENTURE. Monitoring data is collected by the OAM Handler and locally stored. Periodically, e.g., every hour, collected monitoring data is transformed into modelled data and the ANNs are used to predict next period traffic. A workflow is proposed, where the VNTM module solves the VENTURE reconfiguration problem to find the optimal VNT based on the predicted traffic computed by the OAM handler.

We compared the performance of VENTURE through simulation against the static and the threshold-based approaches. We observed savings between $8 \%$ and $42 \%$ in the number of transponders to be installed in the routers when the VENTURE approach was applied. In addition, VENTURE is able to deactivate transponders during low traffic hours thus, decreasing the energy consumption and releasing lightpaths from the underlying optical layer, which contribute to a costs reduction.

Finally, the proposed architecture was experimentally assessed in our SYNERGY test-bed.

\section{ACKNOWLEDGMENT}

The research leading to these results has received funding from the Spanish MINECO SYNERGY project (TEC201459995-R) and from the Catalan Institution for Research and Advanced Studies (ICREA).

\section{REFERENCES}

[1] Ll. Gifre, L. M. Contreras, V. Lopez, and L. Velasco, "Big Data Analytics in Support of Virtual Network Topology Adaptability," in Proc. of the Optical Fiber Communication Conference (OFC), 2016.

[2] F. Morales, M. Ruiz, and L. Velasco, "Virtual Network Topology Reconfiguration based on Big Data Analytics for Traffic Prediction,” in Proc. of the Optical Fiber Communication Conference (OFC), 2016.

[3] L. Velasco, L.M. Contreras, G. Ferraris, A. Stavdas, F. Cugini, M. Wiegand, and J. P. Fernández-Palacios, "A Service-Oriented Hybrid Access Network and Cloud Architecture," IEEE Communications Magazine, vol. 53, pp. 159-165, 2015.

[4] M. Ruiz, M. Germán, L. M. Contreras, and L. Velasco, "Big Databacked Video Distribution in the Telecom Cloud," Elsevier Computer Communications, vol. 84, pp. 1-11, 2016.

[5] D. King and A. Farrel, "A PCE-based Architecture for Application-based Network Operations,” IETF RFC 7491, 2015.

[6] A. Aguado et al., "Dynamic Virtual Network Reconfiguration over SDN Orchestrated Multi-Technology Optical Transport Domains," in Proc. of the European Conference on Optical Communications (ECOC) 2015.

[7] F. Agraz, L. Velasco, J. Perelló, M. Ruiz, S. Spadaro, G. Junyent, and J. Comellas, "Design and Implementation of a GMPLS-Controlled Grooming-capable Optical Transport Network,” IEEE/OSA Journal of Optical Communications and Networking (JOCN), vol. 1, pp. A258A269, 2009.

[8] A. Gençata and B. Mukherjee, "Virtual-Topology Adaptation for WDM Mesh Networks Under Dynamic Traffic," IEEE Transactions on Networking, vol. 11, 2003.

[9] Y. Ohsita, T. Miyamura, S. Arakawa, S. Ata, E. Oki, K. Shiomoto and M. Murata, "Gradually Reconfiguring VNT Based on Estimated Traffic Matrices," IEEE Transactions on Networking, vol. 18, 2010.

[10] N. Fernández, R. Durán, D. Siracusa, A. Francescon, I. de Miguel, E. Salvadori, J. Aguado and R. Lorenzo, "Virtual Topology Reconfiguration in Optical Networks by Means of Cognition: Evaluation and Experimental Validation," IEEE/OSA Journal of Optical Communications and Networking, vol. 7, pp. A162 - A173, 2015.

[11] L. Velasco, A. P. Vela, F. Morales, and M. Ruiz, "Designing, Operating and Re-Optimizing Elastic Optical Networks," IEEE/OSA Journal of Lightwave Technology (JLT), DOI: 10.1109/JLT.2016.2593986, 2016.

[12] J. Boyd, "Destruction and Creation," U.S. Army Command and General Staff College, 1976.

[13] F. Emmert-Streib and M. Dehmer, "Information Theory and Statistical Learning,” Springer Science \& Business Media, 2008.

[14] J.D. Hamilton, "Time Series Analysis," Princeton University Press, 1994.

[15] K. Steiglitz, C. H. Papadimitriou, "Combinatorial Optimization: Algorithms and Complexity,” Prentice-Hall, New Jersey, 1982.

[16] Apache Cassandra: http://cassandra.apache.org/

[17] Apache Spark: http://spark.apache.org/

[18] L. Velasco and Ll. Gifre, "iONE: A Workflow-Oriented ABNO Implementation," in Proc. of the Photonics in Switching Conference, 2015. 